\title{
EL EMPRENDIMIENTO SOCIAL EN EL TURISMO COMUNITARIO DE LA PROVINCIA DE MANABÍ, ECUADOR
}

\author{
Social entrepreneurship in community tourism in the Province of \\ Manabí, Ecuador
}

\section{Carmen Auxiliadora Lucas Mantuano*, Gladys Salazar Olives** y Claudia Katiuska Loor Caicedo***}

\section{RESUMEN}

El sector turismo es considerado en Ecuador como una actividad económica y social, generadora de oportunidades de emprendimiento que impulsan el desarrollo integral e inclusivo y mejoran la calidad de vida de la población. Esta investigación tiene como objetivo analizar el aporte de las Teorías de los Recursos y Capacidades (Grant, 1991; Barney, 1991; Stam, 2009), la del emprendimiento social (Dees, 1998; Pollock, 2015; Buzinde et al., 2017) y la del turismo comunitario (Murphy, 1985; Manyara y Jones, 2007, Kokkranikal y Morrison, 2011) para la creación de empresas turísticas comunitarias en la Provincia de Manabí de Ecuador. La metodología utilizada es de tipo documental y descriptiva. El turismo comunitario en Ecuador, forma parte de una estrategia de desarrollo local, propiciada por el Estado para la reivindicación y autogestión sobre sus territorios y recursos naturales. La Teoría de Recursos y Capacidades proporciona herramientas cognoscitivas a los emprendimientos sociales, a partir de unas relaciones claves entre recursos, capacidades y ventajas competitivas, ésta no se limita sólo al ámbito empresarial sino que por el contrario se pueden extrapolar a diferentes tipos de organizaciones con el fin de mejorar la planeación, control, optimización de sus procesos y consecución de metas y objetivos. Se concluye que la identificación de los recursos internos de los emprendimientos de turismo comunitario permitirá conocer y dimensionar su estructura y así potenciar sus capacidades, sugiriendo un elemento adicional a su autogestión. Es de interés enfatizar

\footnotetext{
* Magister en Tributación y Finanzas. Universidad Laica Eloy Alfaro de Manabí, Facultad de Hotelería y Turismo, Profesor Tiempo Completo Agregado 1. Manta- Ecuador. Correo electrónico: carmen.lucas@uleam.edu.ec https://orcid.org/00000002-4287-2902.

** Magister en Gerencia Educativa. Universidad Laica Eloy Alfaro de Manabí, Campus Pedernales, Profesor Auxiliar Tiempo Completo. $\quad$ Pedernales-Ecuador. $\quad$ Correo electrónico: gladys70salazar@gmail.com https://scolar.google.com.ec/citaciones?user=3p3ciOMAAAAJ\&hl=es\&oi=sao

${ }^{* * *}$ Magister en Enseñanza del idioma inglés. Universidad Laica Eloy Alfaro de Manabí, Facultad de Hotelería y Turismo. Profesor Tiempo Completo Agregado 1. Manta-Ecuador. Correo electrónico: claudia.loor@uleam.edu.ec https://orcid.org/0000-0003-3756-2771
} 
El emprendimiento social en el turismo comunitario de la provincia de Manabí, Ecuador

que el emprendimiento social en el turismo comunitario manabita, requiere de una dimensión económica rentable porque son empresas sociales que poseen una estructura organizativa con sus recursos y capacidades naturales, culturales y humanos que requieren de una inversión económica para su mantenimiento.

Palabras clave: Recursos y capacidades, emprendimiento social, turismo comunitario, Ecuador, emprendimiento turístico.

RECIBIDO: Mayo 2019

ACEPTADO: Julio 2019

\section{ABSTRACT}

The tourism sector is considered in Ecuador as an economic and social activity, generating entrepreneurial opportunities that promote integral and inclusive development and improve the quality of life of the population. The objective of this research is to analyze the contribution of the Theories of Resources and Capacities (Grant, 1991, Barney, 1991, Stam, 2009), social entrepreneurship (Dees, 1998, Pollock, 2015, Buzinde et al., 2017) and that of community tourism (Murphy, 1985; Manyara and Jones, 2007, Kokkranikal and Morrison, 2011) for the creation of community tourism enterprises in the Province of Manabí of Ecuador. The methodology used is documentary and descriptive. Community tourism in Ecuador is part of a strategy of local development, promoted by the State for the claim and self-management of its territories and natural resources. The Theory of Resources and Capacities provides cognitive tools to social enterprises, based on key relationships among resources, capabilities and competitive advantages, this is not limited only to the business sector but on the contrary can be extrapolated to different types of organizations with In order to improve the planning, control, optimization of its processes and consecution of goals and objectives. It is concluded that the identification of the internal resources of community tourism ventures will allow us to know and size their structure and thus enhance their capabilities, suggesting an additional element to their self-management. It is of interest to emphasize that social entrepreneurship in the Manabi community tourism requires a profitable economic dimension because they are social enterprises that have an organizational structure with their natural, cultural and human resources and capacities that require an economic investment for their maintenance.

Keywords: Resources and capacities, social entrepreneurship, community tourism, Ecuador, tourism entrepreneurship.

\section{Introducción}

El potencial turístico de Ecuador se sustenta en la diversidad natural y cultural que posee: riqueza natural, paisajes, sitios históricos, riquezas ancestrales, saberes y costumbres locales, entre otros, constitutivos de espacios turísticos que pueden ser 
aprovechados en la sostenibilidad de la actividad turística y sus respectivos beneficios económicos para el desarrollo integral de la población ecuatoriana.

En este sentido, en la propuesta construida sobre la base del Plan Nacional del Buen Vivir 2013-2017 y en la Estrategia Nacional para el Cambio de la Matriz Productiva, Ecuador consideró el turismo como sector estratégico por su potencialidad, y el Ministerio de Turismo en el año 2016 diseñó políticas de turismo sustentadas en la visión de convertir a Ecuador en un referente turístico de la región, haciendo del turismo un elemento clave para el desarrollo sustentable social y económico (Ministerio de Turismo, 2016).

Asimismo, en el Plan Nacional de Desarrollo 2017-2021 Toda una Vida, Ecuador se propone impulsar el sistema económico y el reconocimiento del país como potencia turística (Senplades, 2017: p. 33), a tal efecto contempla el desarrollo de emprendimientos locales (Senplades, 2017: p. 42), lo que incluye la Zona 4 a la cual pertenece la provincia Manabí junto a la provincia Santo Domingo de los Tsáchilas. Dicho interés nace de los efectos positivos que tiene la generación de una sociedad más emprendedora, implicada en la creación de nuevas actividades económicas.

Frente a esta realidad destaca Morales et al., (2017), tomando como referencia el enfoque y los objetivos en los que se basa el desarrollo de un emprendimiento social, el turismo constituye uno de los sectores que potencian el desarrollo de este tipo de emprendimientos, puesto que las diversas tipologías de turismo tienen el potencial de influir sobre el cambio social (Pollock, 2015; Buzinde et al., 2017), así por ejemplo, el turismo comunitario (Kokkranikal y Morrison, 2011) representa una actividad que puede incidir de forma positiva en el bienestar social y la calidad de vida de las comunidades.

El turismo comunitario se basa en dos aspectos fundamentales: la comunidad y los recursos naturales, lo que hace necesario que los modelos de ventaja competitiva deben ser expandidos para incluir las limitaciones y los desafíos que el medio ambiente natural impone a las empresas, y cómo los recursos y las capacidades interfieren en la interacción de la empresa con su entorno natural.

Por lo antes expuesto, esta investigación centra su estudio en la provincia de Manabí, direccionada por el siguiente objetivo: mostrar cómo desde las Teorías de los recursos y capacidades (TRC), la del emprendimiento social y la del turismo comunitario pueden crearse empresas turísticas comunitarias de carácter dinámico, que se planteen de un modo sostenible el aprovechamiento de los recursos locales y la generación de empleo. La importancia de la misma se expresa como un aporte de la 
El emprendimiento social en el turismo comunitario de la provincia de Manabí, Ecuador

academia universitaria al sector turismo en la modalidad "turismo comunitario", con miras a incentivar el emprendimiento social en el turismo comunitario en la provincia de Manabí de Ecuador, colaborando a su recuperación económica tras el terremoto acaecido el 16 de abril del 2016.

La investigación es de tipo documental y descriptiva, apoyada en la revisión bibliográfica en libros, revistas científicas, documentos oficiales y fuentes virtuales. Se realizó análisis, síntesis y contrastación de los contenidos seleccionados en la puesta en diálogo de las teorías tratadas, para dar respuesta al objetivo central de la investigación. Luego de esta introducción el resto del artículo se organiza de la siguiente manera: una primera parte sobre los fundamentos teóricos de la investigación donde se describe la teoría de los recursos y capacidades, la teoría del emprendimiento social y turismo comunitario, una segunda parte aborda algunos aspectos de la provincia de Manabí en Ecuador y su potencial turístico para el emprendimiento social de turismo comunitario en el contexto de las teorías planteadas, finalmente se cierra con las conclusiones de la investigación.

\section{Fundamentos teóricos}

\section{La Teoría de los recursos y capacidades}

La TRC pertenece al dominio de la estrategia de la organización que asigna importancia a la estructura interna de la misma. Esta teoría fue creada por Penrose postulando que "una empresa es una colección de recursos, un depósito de conocimientos en la medida en que los recursos poseen competencias individuales y son capaces de proporcionar servicios diversos" (Penrose, 1959: p. 27), al mismo tiempo esta teoría describe "los procesos mediante los cuales una empresa crece, conceptualiza a la firma o a la empresa como un conjunto de recursos productivos con organización administrativa" (Sánchez y Herrera, 2016: 135). Teece (1982) amplía la teoría afirmando que las empresas poseen un exceso de recursos los cuales pueden utilizarse con fines de diversificación dando paso a las empresas de multiproducto, y en este sentido agrega Wernerfelt (1984), que la ampliación de los recursos por parte de la empresa agencia el equilibrio de la explotación de los mismos y el desarrollo de otros nuevos. Posteriormente la TRC aumenta su alcance cuando autores como, Barney (1991) y Peteraf (1993) introducen la búsqueda de vínculos entre las capacidades que tienen las organizaciones y su desempeño superior con respecto a sus competidores.

La TRC expresa que en término de recursos y capacidades internas las empresas son heterogéneas, lo que constituye el fundamento de la gestión estratégica (Peteraf, 1993). Por su parte, Barney (2001) expone que la heterogeneidad de las organizaciones se debe a la posesión de recursos: i) valiosos, que deben responder a las amenazas del 


\section{Carmen Lucas Mantuano, Gladys Salazar Olives y Claudia Loor Caicedo}

Telos Vol. 21, No. 3 (2019). 661-680

entorno y aprovechar sus oportunidades; ii) raros o escasos, aquellos que no pueden permitir la obtención de ventajas competitivas con los competidores; iii) recursos difíciles de imitar, sin sustitutos y organizativos, lo que significa que la empresa posea aspectos de orden. Ya Barney (1986), expresa que estas características son aplicables también a los recursos intangibles, lo que le asigna a la empresa poder adquirir una ventaja competitiva sostenida, puesto que los recursos trabajan en conjunto.

Por su parte Stam (2009), advierte que las regiones están dotadas de recursos heterogéneos, en un contexto único, por lo que los emprendimientos en las mismas ofrecen ventajas competitivas sustentables, incluso con productos inimitables que visibilizan y optimizan los valores y la cultura de la localidad.

Los recursos son valiosos porque permiten a las organizaciones desarrollar actividades y pueden considerarse como una fuente competitiva (Porter, 1991). Esta teoría resulta pertinente para utilizarse en economías emergentes por su aporte en la generación de ventajas competitivas sostenibles en el tiempo (West y Bamford, 2005), además de favorecer la realización de estrategias de emprendimiento.

El análisis de los recursos es considerada por sus precursores como el "potencial para extender el entendimiento de la TRC al abordar explícitamente el rol de las acciones de los gestores para estructurar, organizar y aprovechar los recursos de la empresa eficazmente" (Sirmon et al, 2011, p.1390). Por lo que permite analizar cómo las empresas entran en una dinámica de despliegue y utilización de recursos para adaptarse al entorno competitivo predominante, así cada fase da lugar a una capacidad focal específica que se desarrolla a través de acciones centradas en los recursos.

En cuanto a las capacidades, estas son el resultado de implementar, coordinar y combinar un grupo de diferentes recursos, utilizando procesos organizativos para lograr un fin (Grant, 1991). Las capacidades pueden ser de dos tipos: operativas y dinámicas. Las operativas se refieren a la ejecución de actividades y sus respectivas rutinas. Las dinámicas son aquellas que implican adaptación y cambio para construir, integrar o reconstruir otros recursos y capacidades (Helfat y Peteraf, 2009). Por su parte, Pérez y Toro (2015), a las capacidades dinámicas las denominan de absorción por estar conformadas por la adquisición y asimilación de conocimiento externo e interno, lo cual permite la creación de valor mediante el desarrollo de habilidades y destrezas.

La principal tendencia de la TRC se encuentra en la investigación en torno a las capacidades. Se asocian éstas al desempeño de la empresa (Mithas et al, 2011; Camisón y Villar-López, 2014; Lin y Wu, 2014), la construcción de capacidades críticas para el aprovechamiento de los recursos que permitan el éxito de la empresa (Ulaga y Reinartz, 
El emprendimiento social en el turismo comunitario de la provincia de Manabí, Ecuador

2011), y su desarrollo para la sustentabilidad (Dao et al, 2011). Prevalece además el avance del concepto conocimiento en los procesos de gestión, dinámica del ajuste y microfundamentos.

De lo anteriormente expuesto, se hace énfasis que para que la empresa permanezca en el mercado y pueda obtener beneficios a largo plazo, debe elaborar y entregar constantemente productos y/o servicios innovadores a los clientes. Todo ello a través de una gestión estratégica con una rutina organizativa con interacciones establecidas entre los recursos y capacidades, de tal forma, que dado los cambios constantes en el mercado, se posibilite la adaptabilidad de la empresa.

\section{La Teoría del emprendimiento social y el turismo Comunitario}

La teoría del emprendimiento social se deriva de la teoría del emprendimiento (Dees, 1998; Sullivan et al, 2003; Austin et al, 2006; Chell, 2007; Martin y Osberg, 2007), y ambas aún no tienen una definición clara del concepto, prestándose a confusiones en la práctica. En esta investigación se exponen algunas definiciones en orden cronológico.

Para Fowler (2000), el emprendimiento social es la creación de estructura, relaciones, instituciones, organizaciones y prácticas socioeconómicas viables que generan y sostienen beneficios sociales. Por su parte, Johnson (2000) define el emprendimiento social como un enfoque innovador para hacer frente a las necesidades sociales complejas, con su énfasis en la resolución de problemas y la innovación social. El Canadian Centre for Social Entrepreneurship Group (2001), lo define como iniciativas innovadoras con la doble finalidad de asegurar que la inversión genere tanto rentabilidad social como económica, desarrolladas tanto por sectores privados como públicos y voluntarios. Según MacMillan (2003), comprende el proceso mediante el cual la creación de una nueva empresa de negocios conduce a la mejora de la riqueza social y el beneficio empresarial.

En este mismo orden de ideas, Shaw (2004), define el emprendimiento social como el trabajo de las organizaciones comunitarias, voluntarias, públicas y privadas con fines de desarrollo social y no solo para obtener beneficios económicos. También el emprendimiento social abarca las actividades y procesos realizados para descubrir, definir y aprovechar las oportunidades que lleven al mejoramiento de la riqueza social mediante la creación de nuevas empresas o la gestión de las organizaciones existentes de un modo innovador (Zahra et al., 2009).

Por su parte la organización Said Business School (2018), expresa que el emprendimiento social es la práctica de combinar la innovación, el ingenio y la oportunidad de abordar los desafíos sociales y ambientales críticos, transformando los 


\section{Carmen Lucas Mantuano, Gladys Salazar Olives y Claudia Loor Caicedo}

Telos Vol. 21, No. 3 (2019). 661-680

sistemas y prácticas que son las causas fundamentales de la pobreza, la marginación, el deterioro ambiental y la consiguiente pérdida de la dignidad humana. Esta actividad puede hacerse con fines o sin fines de lucro, siendo en ambos casos el objetivo principal la creación de cambios sostenibles en los sistemas.

Según Marín y Rivera (2014), las empresas que nacen del emprendimiento social son producto de una iniciativa de los individuos por y para la comunidad, conjuga elementos de desarrollo humano y formación integral, en pleno respeto de la identidad y autodefinición de las personas y comunidades.

Estas definiciones muestran que el emprendimiento social tiene dos dimensiones: una dimensión social que crea valor social y una dimensión económica que busca el beneficio económico. Lo interesante del asunto es, que si de lo que se trata con el emprendimiento social es crear empresas para la generación de empleo, debe haber un equilibrio entre las dos dimensiones para que los empleados experimenten un cambio económico con impacto social: el beneficio económico se traduce en transformación social positiva diferencia del concepto tradicional de emprendimiento que hace énfasis en el rendimiento privado.

Con respecto al concepto de turismo comunitario quien primero lo plantea es Murphy (1985), donde analiza aspectos relacionados con el turismo y las áreas rurales de los países menos adelantados, de forma complementaria Maldonado (2005), plantea que el turismo comunitario tiene como objetivo la preservación de la identidad étnica, la valoración y transmisión del patrimonio cultural en todas sus formas, destaca que las culturas autóctonas son portadoras de valores, de historia y de identidad. Por su parte, Manyara y Jones (2007), plantean el turismo comunitario como un sector de impacto sobre las familias de la población local, el desarrollo de la región y los estilos de vida.

El turismo comunitario constituye una modalidad del Turismo rural, a la vez que se integra al modelo de desarrollo turístico del país. En la práctica del turismo comunitario, la especificidad más importante es el control del producto turístico por parte de la comunidad o de las comunidades. De este modo, todas las decisiones en torno al proceso de desarrollo del turismo comunitario serán adoptadas por la comunidad y serán dirigidas a la generación de empleos con ingresos económicos justos, además y con esencial importancia, contribuir a la conservación del medio ambiente (Inostroza, 2008).

El turismo comunitario está considerado como una forma de emprendimiento social, que asiste al desarrollo integral de las comunidades receptoras, en aras de obtener relaciones de calidad y desarrollo humano entre visitantes y locales (Cardona y Burgos, 
El emprendimiento social en el turismo comunitario de la provincia de Manabí, Ecuador

2015), asimismo requiere que la comunidad involucrada en la gestión, desarrolle capacidades de auto-organización y acción colectiva, relaciones cara a cara, relaciones socio-culturales y relaciones socio-políticas que permeen la interacción entre la comunidad, el mercado y el Estado para lograr efectividad en la gobernanza.

\section{La provincia de Manabí de Ecuador y su potencial turístico para el emprendimiento social del turismo comunitario}

Atendiendo a los datos geográficos proporcionados por el Consejo Provincial de Manabí fechada el jueves 21 de febrero del 2019, se tiene que la provincia Manabí se encuentra situada en la costa ecuatoriana, en el emplazamiento centro-noroeste de Ecuador, limitando por el norte con la provincia Esmeraldas, al sur con las provincias Santa Elena y Guayas, al este con las provincias Guayas, Los Ríos y Santo Domingo de los Tsáchilas, y por el oeste con el Océano Pacífico, el cual baña 350 kilómetros de costa manabita (Consejo Provincial de Manabí, 2019).

La naturaleza dotó el territorio manabita de recursos naturales diversos atrayentes para el turismo tanto para propios como para extraños, y de recursos culturales de importantes atractivos. Por ser una provincia costeña cuenta con extensas playas (cantones: Jama, Jaramijó, Jipijapa, Manta, Montecristi, Pedernales, Portoviejo, Puerto López, San Vicente y Sucre), balnearios de agua dulce y cascadas (cantones: Bolívar, El Carmen, Chone, Flavio Alfaro, Jama, Jaramijó, Jipijapa, Junín, Manta, Montecristi, Olmedo, Paján, Pedernales, Pichincha, Portoviejo, Puerto López, Rocafuerte, Santa Ana, Tosagua, 24 de Mayo y San Vicente), Islas e Islotes ( Montecristi, Puerto López y Sucre), Estuarios (cantones: Chone/Sucre, Pedernales, Portoviejo y Puerto López) y reservas naturales: áreas protegidas (bosques protectores y senderos) y cerros/colinas (cantones: Bolívar, El Carmen, Chone, Jama, Jaramijó, Jipijapa, Junín, Manta, Montecristi, Paján, Pedernales, Pichincha, Portoviejo, Puerto López y San Vicente) (Álava, 2018).

A todos estos recursos naturales se le suman la belleza paisajística, la diversidad de la flora y fauna manabita, las fincas y espacios rurales que posibilitan actividades turísticas. En lo atinente a los atractivos culturales, la provincia Manabí cuenta con monumentos históricos, museos, iglesias, plazas, comercio, infraestructura hotelera y una gastronomía variada, entre otros atractivos; que si bien el turismo comunitario se centra en los recursos naturales, también muchos de los recursos culturales requieren de preservación y pudieran dar origen a emprendimientos comunitarios donde se complementen recursos naturales y culturales que fomenten la generación de riqueza a través del empleo a la población manabita. 
Declarados los atributos turísticos naturales y culturales de la provincia Manabí, es de interés pasar a las interrelaciones teóricas para construir una nueva teoría que integre la de recursos y capacidades, la del emprendimiento social y la de turismo comunitario, cuya finalidad se fundamenta en la creación de nuevas empresas de emprendimiento turístico comunitario en Manabí, o en su defecto emprendimientos donde se innove en las empresas ya existente; de cualquier modo, de lo que se trata es de promover el emprendimiento social en el turismo comunitario de la provincia Manabí, asomando acciones, estrategias y políticas dirigidas al gobierno del Estado, al gobierno municipal, al sector productivo y a la población manabita; para la creación de empresas de turismo comunitario generadoras de empleo.

En los dos últimos Planes de Desarrollo Nacional en Ecuador (2013-2017 El Buen Vivir y el 2017-2021 Toda una Vida) se ha previsto el desarrollo sostenible, declarado en el Plan 2013-2017 (Senplades, 2013) en los objetivos 7: Garantizar los derechos de la naturaleza y promover la sostenibilidad ambiental territorial y global (p.221) y el 8: Consolidar el sistema económico social y solidario, de forma sostenible (p.247). Así mismo, el Plan 2017-2021 (Senplades, 2017) declara el desarrollo sostenible en el Eje 2 Economía al Servicio de la Sociedad, objetivos 4: Consolidar la sostenibilidad del sistema económico social y solidario, y afianzar la dolarización (p. 76) y 5: Impulsar la productividad y competitividad para el crecimiento económico sostenible de manera redistributiva y solidaria (p.80).

La discusión expuesta en la literatura científica sobre lo sostenible y lo sustentable es amplia y explican muy bien el recorrido transformador de estos conceptos estrechamente relacionados; de modo que no puede haber sostenibilidad de un sistema económico sin que haya una sustentabilidad en el crecimiento económico expresado en términos de desarrollo versus equilibrio del ecosistema, lo que implica mantener una elevada capacidad productiva y proteger a la vez los recursos naturales, lo que no sería posible si no sabemos cuánto tenemos que conservar y de qué manera, y por esta causa, de todos depende que el desarrollo sea sostenido y sustentable (Boada y Toledo, 2003).

El fomento del emprendimiento social en el turismo de Manabí es posible hacerlo realidad porque Ecuador cuenta con la normativa legal necesaria que garantiza el estado de derecho generador de confianza en la población empresaria: Asamblea Constituyente (2008), Ministerio de Turismo (2002), la Planificación Nacional para el Desarrollo, los Reglamentos de las Instituciones locales, entre otros; pero privilegiadamente con los recursos naturales, culturales y humanos fundamentales para tal fin. 
El emprendimiento social en el turismo comunitario de la provincia de Manabí, Ecuador

Ecuador es pionero en América Latina en turismo comunitario (Ruíz y Solis, 2007), el cual viene realizando desde mediados de la década de los años 80. El mismo surgió como una alternativa económica de las propias comunidades campesinas, indígenas y afro ecuatorianas, tanto para obtener ingresos adicionales como para contribuir a la revalorización y defensa de los recursos naturales locales.

Es a partir del 2002 cuando la Ley de Turismo del Ecuador, reconoce a "las comunidades como actores diferenciados del sector, desatando desde entonces un proceso complejo y disputado de diseño de la política de turismo comunitario" (Unkuch y Rodrigues de Caires, 2017: p. 60).

No obstante, Solis (2007), destaca los desafíos básicos comunes propuestos por el Estado: la calidad, la conectividad, la dotación de servicios básicos, la diversificación de los productos ofertados, y el acceso a mercados y conocimientos; no se cumplieron en su totalidad. Esto pudo haberse dado porque la actividad turística comunitaria de Ecuador, desde sus inicios tuvo una debilidad en la dimensión socio institucional, por lo que consideran Unkuch y Rodrigues de Caires (2017), que las políticas en este sector de la economía para que resulten efectivas van a depender de una adecuada interacción entre la comunidad, el mercado y el Estado; como un apropiado arreglo de gobernanza

En efecto, el turismo comunitario en Ecuador, forma parte de una estrategia de desarrollo local y de consolidación política de la comunidad, propiciada por el Estado para la reivindicación y autogestión sobre sus territorios y recursos naturales locales. Así lo declara el Ministerio de Turismo (2002), mediante la Ley de Turismo del Ecuador publicada en el Registro oficial el 22 de diciembre del 2002 (con su última modificación emitida el 29 de diciembre del 2014). Y también la Asamblea Constituyente (2008) mediante la Constitución de la República del Ecuador de 2008 (Montecristi) publicada en el Registro Oficial el 20 de octubre del 2008 (con modificaciones en el 2011, 2014 y 2018).

En el mismo orden de ideas, el Plan Nacional del Buen Vivir 2013-2017 declaró entre sus grandes objetivos nacionales "Mejorar la calidad de vida de la población", y contempla como lineamiento el "fomentar el turismo comunitario y turismo social", con la finalidad de alcanzar el objetivo de "Consolidar el sistema económico social y solidario, de forma sostenible" (Senplades, 2017).

Por su parte el Plan Nacional de Desarrollo 2017-2021 (Senplades, 2017) considera los recursos naturales como base de la economía nacional (p.38) y establece el mejoramiento de la infraestructura productiva, la tecnología y el conocimiento que la población del territorio rural, en este caso, aplicado a la que la provincia de Manabí requiere para fortalecer los emprendimientos turísticos comunitarios existentes, pero 


\section{Carmen Lucas Mantuano, Gladys Salazar Olives y Claudia Loor Caicedo}

Telos Vol. 21, No. 3 (2019). 661-680

sobre todo para incentivar la inversión en la creación de nuevas empresas de turismo comunitario y social.

La TRC desde su origen ha estado asociada a los recursos heterogéneos de la empresa privada y su desarrollo, definiéndose la empresa en términos de sus recursos productivos que le generan una ventaja competitiva sustentable. No obstante, la orientación de esta teoría asociada al emprendimiento social (Schwab Foundation, 2018) propende el desarrollo comunitario sustentable, por lo que la comunidad manabita puede convertirse en una empresa innovadora que impulse el emprendimiento social en el turismo comunitario para obtener bienestar socioeconómico, y así, disminuir la pobreza y evitar la migración. De este modo, bajo una visión macro transformadora, la comunidad puede contribuir al desarrollo endógeno y sustentable de una nación. $\mathrm{Y}$ aunque estas iniciativas "responden a realidades inmediatas o locales, los impactos son de alcance global [pero] se requieren mecanismos de equilibrio e inclusión" (Ortega-Martínez, 2018: p. 92).

La TRC proporciona herramientas cognoscitivas a los emprendedores para recalcar que sus recursos productivos le generan una ventaja competitiva sustentable (Stam, 2009; Schwab Foundation, 2018), y también le permiten obtener ventajas competitivas sostenibles en el tiempo (West y Bamford, 2005). En el cuadro No 1, tomando el referente teórico de Grant (1991), Suárez e Ibarra (2002) y Sánchez y Herrera (2016), se presentan unas relaciones claves entre recursos, capacidades y ventajas competitivas, que pueden adaptarse para la generación de estrategias en emprendimientos de turismo comunitario, caracterizando elementos claves en cada fase de modo general, que dependerán de la especificidad de la unidad de estudio.

En primera instancia se clasifica los recursos en tangibles, intangibles y humanos. A partir del empleo conjunto de los activos tangibles e intangibles que posee la empresa en combinación con su conocimiento tecnológico y organizativo, éste despliega diversas capacidades operativas (aprovisionamiento, producción, distribución), innovadoras o directivas. Las capacidades están ligadas al capital humano, se apoyan sobre todo en los activos intangibles, particularmente en el conocimiento tecnológico y organizativo de la empresa, son capaces de gobernar la transformación de los insumos en productos o servicios finales, generan valor añadido y determinan la eficiencia y el grado de innovación de la entidad. No sólo dependen de la base de activos de la empresa, sino también de su propia habilidad para combinarlos, integrarlos y movilizarlos a través de diversas rutinas organizativas (Grant, 1991). 
El emprendimiento social en el turismo comunitario de la provincia de Manabí, Ecuador

Le sigue una evaluación referente al potencial de la generación de renta de los recursos y capacidades, de modo tal que pueda precisar las posibilidades que tiene el turismo comunitario para crear, mantener o incrementar una ventaja competitiva. La evaluación va a permitir diseñar una estrategia que agencie el aprovechamiento eficiente de recursos y capacidades de forma pertinente en función con el entorno. Al poner en ejecución la estrategia, se reinicia el proceso de análisis para garantizar la mejora continua en la producción y lograr un desarrollo sostenido. El proceso de análisis estratégico va a constituirse en un círculo virtuoso que instaura la dinámica y la creatividad para la innovación y el emprendimiento empresarial.

\section{Cuadro No 1}

Enfoque de La Teoría de Recursos y Capacidades en el turismo comunitario

\begin{tabular}{|c|c|c|}
\hline Fase de identificación & $\begin{array}{l}\text { Recursos } \\
\text { (Identificar los recursos } \\
\text { del emprendimiento) } \\
\\
\text { Capacidades } \\
\text { ¿Qué puede hacer el } \\
\text { emprendimiento? }\end{array}$ & 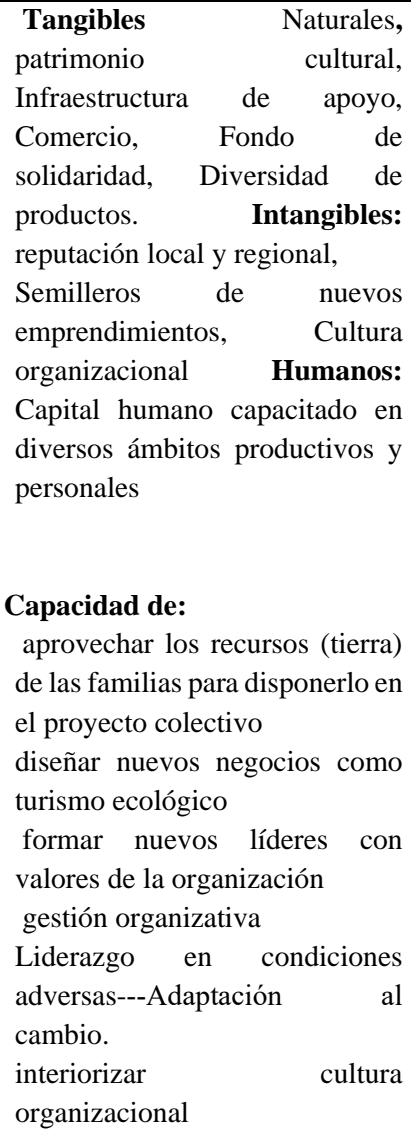 \\
\hline
\end{tabular}




\begin{tabular}{|c|c|c|}
\hline & & $\begin{array}{l}\text { consolidar alianzas con otros } \\
\text { estamentos }\end{array}$ \\
\hline Fase de Evaluación & $\begin{array}{l}\text { Evaluar los recursos y } \\
\text { capacidades } \\
\text { términos de su potencial } \\
\text { para crear, mantener y } \\
\text { desarrollar una ventaja } \\
\text { competitiva }\end{array}$ & 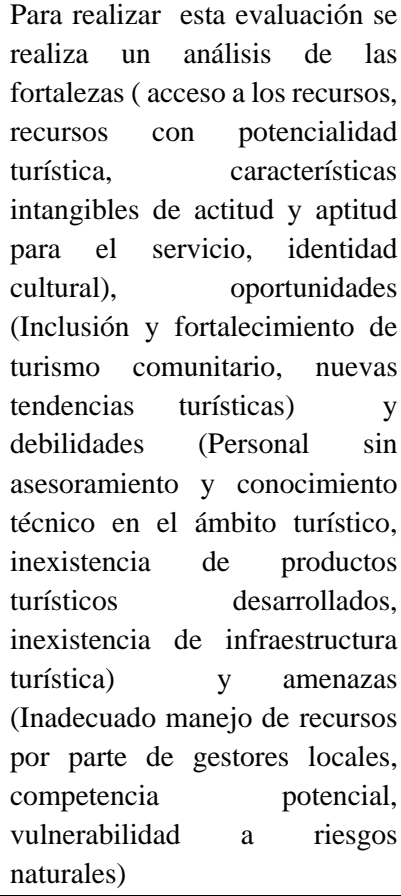 \\
\hline Fase de diseño & Estrategias & $\begin{array}{l}\text { El diseño y puesta en marcha de } \\
\text { una estrategia de organización a } \\
\text { la luz de potenciar los recursos } \\
\text { con los que cuenta y las } \\
\text { capacidades alcanzadas al } \\
\text { momento con el uso y } \\
\text { disposición de estos activos, por } \\
\text { lo que se hace necesario la } \\
\text { reflexión en torno al potencial de } \\
\text { crecimiento y expansión. }\end{array}$ \\
\hline
\end{tabular}

Fuente: Elaboración propia adaptado de Grant (1991); Suárez e Ibarra (2002), Sánchez y Herrera (2016) 
El emprendimiento social en el turismo comunitario de la provincia de Manabí, Ecuador

Destaca Vásquez y Manzanilla (2018), la ventaja competitiva no debe ser enfocada únicamente como que cada empresa deba ser capaz de crear valor para sus compradores, sino que se debe de considerar las limitaciones y los desafíos que el medio ambiente natural impone, ya que éstas, interactúan con sus recursos, las capacidades y su entorno natural y así generar una ventaja competitiva para comunidades heterogéneas.

La actividad de la búsqueda de innovación en algunos procesos con recursos que resultasen idóneos para la diversificación puede asignarse a algunos miembros de la empresa social turística comunitaria manabita, o en su defecto hacer asociaciones con especialistas académicos, técnicos de la misma comunidad, con fines de lucro o sin fines de lucro según acuerdos entre las partes. Ello con la finalidad de reconocer que la innovación social es la base de todo emprendimiento social, el cual lleva a la resolución de problemas y a la generación de rentabilidad social y económica para coadyuvar en la erradicación de la pobreza en Manabí cuyos niveles de pobreza multidimensional están por encima del promedio nacional (Senplades, 2017: p. 113).

También el análisis estratégico las empresas turísticas comunitarias de Manabí pueden hacerlo en conjunto. Esta modalidad les ayuda a identificar sus fortalezas, debilidades y necesidades en sus capacidades operativas y dinámicas, de modo que introduzcan vínculos entre las capacidades con las que cuentan para competir con otras empresas externas de su localidad y con el turismo de otros países. Ello puede hacerse con capital propio, con el apoyo del gobierno local o de capital externo, pudiendo constituir circuitos comerciales solidarios y/o encadenamientos productivos. Todo esto se facilita cuando se trata de empresas que cosechan en sus tierras los recursos que le proporcionan la materia prima para la elaboración de productos, y Manabí cuenta con estos atributos.

Manabí tiene recursos y capacidades tanto naturales como culturales y vivenciales para el desarrollo del turismo comunitario social, un desarrollo local turístico que requiere de "servicios e instalaciones que posibiliten los desplazamientos humanos, para ello es necesario mejorar las condiciones de vida de los manabitas, logrando un equilibrio entre lo económico, social y ambiental, y satisfaciendo las necesidades de los habitantes y visitantes" (Arroyo, 2018: p. 47).

\section{Conclusiones}

El turismo comunitario de emprendimiento social en la provincia Manabí de Ecuador, constituye un rubro muy importante para la generación de empleo, la redistribución de la riqueza, empoderar a las familias emprendedoras, fomentar en la población relaciones equitativas y respetuosas, contribuir a la conservación de los 
recursos naturales y culturales, y favorecer el desarrollo sostenible de la provincia. Para el cumplimiento de esta misión social, las empresas comunitarias de emprendimiento social, han de tener una dimensión económica rentable, de modo que sus empleados (socios comunitarios) hayan mejorado su nivel de vida y se sientan suficientemente motivados para satisfacer las exigencias que impone el usuario del turismo internacional en el siglo XXI.

La TRC no se limita sólo al ámbito empresarial sino que por el contrario se pueden extrapolar a diferentes tipo de organizaciones, con el fin de mejorar la planeación, control, optimización de sus procesos y consecución de metas y objetivos. La identificación de los recursos internos permite conocer y dimensionar su estructura y así potenciar sus capacidades, sugiriendo un elemento adicional a su autogestión, en el que soportar sus prácticas en busca de la satisfacción óptima de las expectativas de todas y cada una de sus asociados y la comunidad en la que repercute.

Es de interés enfatizar que el emprendimiento social en el turismo comunitario manabita, requiere de la dimensión económica rentable porque son empresas sociales que poseen una estructura organizativa con sus recursos y capacidades naturales, culturales y humanos que requieren de una inversión económica para su mantenimiento, siempre aplicando principios de equidad entre los miembros de la comunidad; además como toda estructura organizativa posee estrategias, normas y valores que hacen posible su funcionalidad, competitividad y sostenibilidad en el mercado turístico.

Como sugerencia a los futuros emprendedores sociales del turismo comunitario en Manabí, es recomendable que tengan un registro de los emprendimientos sociales comunitarios y apliquen el marco práctico a nivel de análisis estratégico, para que precisen los recursos y competencias con las que cuentan y su respectivo entramado organizativo, de modo que puedan tomar decisiones pertinentes con las oportunidades, y teniendo como norte que la empresa social de turismo comunitario a crear tenga dos dimensiones amalgamadas: una económica y otra social para generar empleos dignos que eleven el nivel de vida de la población manabita.

\section{Referencias bibliográficas}

Álava, María (2018). El turismo en la reactivación económica de la provincia de Manabí, Año 2017. Tesis de Maestría en Finanzas y Economía Empresarial. Universidad Católica de Santiago de Guayaquil. Ecuador.

Arroyo, María (2018). Importancia del turismo para el desarrollo local de la provincia Manabí. Revista electrónica Cooperación Universidad Sociedad. Volumen 3, No 1. Ecuador (Pp. $44-49$ ). 
El emprendimiento social en el turismo comunitario de la provincia de Manabí, Ecuador

Asamblea Constituyente (2008). Constitución de la República del Ecuador. Publicada en el Registro Oficial el 20 de octubre del 2008 (con modificaciones en el 2011, 2014 y 2018). Ecuador.

Austin, James, Stevenson, Howar y Wei-Skillern, Jane (2006).Social and commercial entrepreneurship: The same, different or both? Entrepreneurship Theory and Practice. Volumen 47, No 3. Brasil (Pp. 370-384).

Barney, Jay (1986). Strategic factor markets: expectations, luck, and business strategy. Management Science. Volumen 32, No 10. Estados Unidos de Norteamérica (Pp. 1231-1241).

Barney, Jay (1991). Firm resources and sustained competitive advantage. Journal of Management. Volumen 17, No 1. Estados Unidos de Norteamérica (Pp. 99120).

Barney, Jay (2001). Is the resource-based "view" a useful perspective for strategic management research? Yes. Academy of Management Review. Volumen 26, No 1. Estados Unidos de Norteamérica (Pp. 41-56).

Boada, Martí y Toledo, Víctor (2003). El planeta nuestro cuerpo. La Ecología, el ambientalismo y la crisis de la modernidad (primera edición). Editorial Fondo de Cultura Económica. México.

Buzinde, Christine; Shockeley. Gordon; Anderson Kathleen; Edward, Dee; Frank Peter (2017).Theorizing Social Entrepreneurship Within Tourism Studies. Social Entrepreneurship and Tourism. Springer. Estados Unidos de Norteamérica.

Camisón, Cesar y Villar-López, Ana (2014) Organizational Innovation as an Enabler of Technological Innovation Capabilities and Firm Performance. Journal of Business Research.No 67. Países Bajos (Pp. 2891-2902).

Canadian Centre for Social Entrepreneurship (2001). Social Entrepreneurship Discussion Extraído http://citeseerx.ist.psu.edu/viewdoc/download?doi=10.1.1.194.4683\&rep=rep1 \&type=pdf consulta: 05/01/19.

Cardona, Martha y Burgos, Rosalía (2015). El turismo comunitario en Colombia: iniciativa de desarrollo local y estrategia de empoderamiento del patrimonio cultural. Administración y Desarrollo. Extraído de https://dialnet.unirioja.es/descarga/articulo/6403410.pdf consulta: 09-04-19.

Chell, Eizabeth (2007). Social enterprise and entrepreneurship: towards a convergent theory of the entrepreneurial process. International Small Business Journal. Volumen 25, No 1. Estados Unidos de Norteamérica (Pp. 5-26).

Consejo Provincial de Manabí (2019). Datos Geográficos. Extraído de http://www.manabi.gob.ec/institucion consulta: 15/01/19.

Dao, Viet.; Langella, Ian., y Carbo, Jerry (2011). From green to sustainability: Information Technology and an integrated sustainability framework. Journal of Strategic Information Systems, Volumen 20, No 1. Países Bajos (Pp. 63-79).

Dees, Gregory (1998). The meaning of social entrepreneurship. Comments and suggestions contributed from the Social Entrepreneurship Funders Working 
Group, 6. Extraído de https://entrepreneurship.duke.edu/news-item/themeaning-of-social-entrepreneurship/ consulta (04/11/2018).

Fowler, Alan (2000). NGDOs as a moment in history: beyond aid to social entrepreneurship or civic innovation? Third World Quarterly. Volumen 21, No 4. Reino Unido (Pp 637-656).

Grant, Robert (1991). The resource based theory of competitive advantage implication for strategy formulation. California Management Review. Volumen 33, No 3. Estados Unidos de Norteamérica (Pp 114-135).

Helfat, Costance y Peteraf, Margaret (2009). Understanding dynamic capabilities: progress along a developmental path. Strategic Organization. Volumen 7, No 1. Estados Unidos de Norteamérica (Pp 91-102).

Inostroza, Gabriel (2008). Aportes para un modelo de gestión sostenible del turismo comunitario en la Región Andina. Revista Gestión Turística. No 10, Ecuador (Pp.77-90).

Johnson, Sherill (2000). Literature review on social entrepreneurship. Canadian Center for Social Entrepreneurship. Extraído de https://www.researchgate.net/publication/246704544_Literature_Review_Of_S ocial_Entrepreneurship consulta 11/02/19.

Kokkranikal, Jithendran.; Morrison, Alison (2011). Community networks and sustainable livelihoods in tourism: The role of entrepreneurial innovation. Tourism Planning and Development. Volumen 8, No 2. Reino Unido (Pp 137156).

Lin, Yini, y Wu, Lei-Yu (2014). Exploring the role of dynamic capabilities in firm performance under the resource-based view framework. Journal of Business Research, Volumen 67, No 3. Países Bajos (Pp 407-413).

MacMillan, Ian (2003). Social entrepreneurs: playing the role of change agents in society. Extraído de: https://knowledge.wharton.upenn.edu/article/socialentrepreneurs-playing-the-role-of-change-agents-in-society/ consulta 15/02/19.

Maldonado, Carlos (2005). Pautas metodológicas para el análisis de experiencias de turismo comunitario. Oficina Internacional del Trabajo (OIT). Suiza.

Manyara, Geofrey y Jones, Eleri (2007). Community-based Tourism Enterprises Development in Kenya: An exploration of their potential as avenues of poverty reduction. Journal of Sustainable Tourism. Volumen 15, No 6. Reino Unido (Pp. 628-644).

Marín, Aramís y Rivera, Igor (2014). Revisión teórica y propuesta de estudio sobre el emprendimiento social y la innovación tecnológica. Acta Universitaria Volumen 24, No. 1. México (Pp. 48-58).

Martin, Roger y Osberg, Sally (2007). Social entrepreneurship: The case for definition. Stanford Social Innovation Review. Volumen 80, No 4. Estados Unidos de Norteamérica (Pp. 252-283). 
El emprendimiento social en el turismo comunitario de la provincia de Manabí, Ecuador

Ministerio de Turismo (2002).Ley de Turismo del Ecuador. Ley 97. Registro Oficial Suplemento 733 de 27/12/02. Última modificación: 29/12/14. Ecuador.

Ministerio de Turismo (2016). Rendición de Cuentas 2016. Informe Narrativo Rendición de Cuentas 2016. Dirección de Seguimiento de Planes, Programas y Proyectos. Ecuador.

Mithas, Sunil; Ramasubbu, Narayan; and Sambamurthy, Vallabhajosyula (2011). "How Information Management Capability Influences Firm Performance." MIS Quarterly. Volumen 35, No 1. Estados Unidos de Norteamérica (Pp.237-256).

Morales, Ximena; Morales, Diana; Hong Eduardo (2017). Emprendimiento social en el sector del turismo comunitario: una primera aproximación al caso de las comunidades indígenas del Ecuador. Revista Iberoamericana de TurismoRITUR. Extraído de http://www.seer.ufal.br/index.php/ritur/article/view/3322 consulta: 16-05-19.

Murphy, Peter (1985). Tourism: A community approach. Editorial Routledge. Estados Unidos de Norteamérica.

Ortega-Martínez, Rosa (2018). Emprendimiento Social: de la caridad al empoderamiento. Extraído de https://www.ecorfan.org/bolivia/series/Estudio_del_emprendimiento_contemp oraneo_en_America_Latina/Estudio_del_emprendimiento_contempor\%C3\%A 1neo_en_America_Latina_8.pdf consulta 05/02/19.

Penrose, Edith (1959) The Theory of the Growth of the Firm. En Foss, Resources, Firm and Strategies. Editorial John Wiley. Estados Unidos de Norteamérica.

Pérez, Elkin y Toro, Ivan (2015). Capacidades de absorción: ¿dónde estamos ahora y dónde queremos estar en el futuro?. Revista Espacios. Extraído de https://www.revistaespacios.com/a15v36n11/15361101.html consulta: 08/01/19

Peteraf, Margaret (1993). The conerstones of competitive advantage: A resource-based view. Strategic Management. Volumen 14, No 3. Estados Unidos de Norteamérica (Pp. 179-191).

Pollock, Anna (2015). Social entrepreneurship in tourism: The conscious travel approach. Extraído de http://www.tipse.org/wpcontent/uploads/2015/07/conscious_tourism_publication.pdf consulta: 08/01/19

Porter, Michael (1991). Towards a dynamics theory or strategy. Management Journal. Volumen 12. Estados Unidos de Norteamérica (Pp. 95-117).

Ruíz, Esteban y Solis, Doris (2007). Turismo comunitario en Ecuador: Desarrollo y sostenibilidad social. Coordinado por Esteben Ruíz y Doris Solis, pp. 29-50. Abya-Yala/Universidad de Cuenca. Ecuador:

Said Business School (2018). Who are Social Entrepreneurs? Extraído de:

https://www.sbs.ox.ac.uk/research/skoll-centre-social-entrepreneurship/who-aresocial-entrepreneurs consulta: 12/01/19

Sánchez, Sandra y Herrera, Margarita (2016). Los recursos humanos bajo el enfoque de la Teoría de los recursos y capacidades. Revista de la Facultad de Ciencias 
Económicas: Investigación y Reflexión. Volumen XXIV, No 2. Colombia (Pp.133-146).

Schwab Foundation (2018). Social entrepreneurship. Retrieved from. Extraído de: https://www.schwabfound.org/what-is-social-entrepreneurship consulta: 05/01/19.

Senplades (2013). Plan Nacional de Desarrollo 2013-2017 Todo el mundo mejor/Plan Nacional para el Buen Vivir 2013-2017. Aprobado el 24 de junio de 2013, Resolución N ${ }^{\circ}$ CNP-002-2013. Ecuador

Senplades (2017). Plan Nacional de Desarrollo 2017-2021 Toda una Vida. Aprobado el 22 de septiembre de 2017, Resolución N ${ }^{\circ}$ CNP-003-2017. Ecuador: Secretaría Nacional de Planificación y Desarrollo. Ecuador.

Shaw, Eleanor (2004). Marketing in the social Enterprise context: is it entrepreneurial? Qualitative Marketing Research: an International Journal. Volumen 7, No 3. Reino Unido (Pp.194-205).

Sirmon, David; Michael, Hitt; Duane, Ireland y Brett, Gilbert (2011). Resource Orchestration to Create Competitive Advantage: Breadth, Depth, and Life Cycle Effects. Journal of Management. Volumen 37, No 5. Estados Unidos de Norteamérica. (Pp. 1390-1412).

Solis, Doris (2007). De la resistencia a la sostenibilidad: el proceso histórico del Turismo Comunitario en Ecuador y sus retos actuales. En Turismo Comunitario en Ecuador: desarrollo y sostenibilidad social. Coordinado por Esteben Ruíz y Doris Solis. Abya-Yala/Universidad de Cuenca. Ecuador.

Stam, Erik (2009) Entrepreneurship, Evolution and Geography. Revista Papers on Economics and Evolution. Extraído de https://pdfs.semanticscholar.org/11cc/a75fe9ef7dbcd52bdd1662e106f274c18d 24.pdf consulta: 08/02/19.

Suárez, Jesús e Ibarra, Santiago (2002). La teoría de los recursos y capacidades. Un enfoque de la estrategia empresarial. Revista Anales de estudios económicos y empresariales. Número 15. España (Pp. 63-89).

Sullivan, Gillian, Weerawardena, Jay y Carnegie, Kashonia (2003). Social entrepreneurship: Towards conceptualization. International. Journal of Nonprofit and Voluntary Sector Marketing. Volumen 8, No 1. Estados Unidos de Norteamérica (Pp. 76-88).

Teece, David (1982). Towards an economic theory of the multiproduct firms. Journal of Economic Behavior \& Organization. Volumen 3, No 1. Paises Bajos (Pp.39-63).

Ulaga, Wolfgang y Reinartz, Wener. J. (2011). Hybrid offerings: How manufacturing firms combine godos and services successfully. Journal of Marketing. Volumen 75, No 6. Estados Unidos de Norteamérica (Pp. 5-23). 
El emprendimiento social en el turismo comunitario de la provincia de Manabí, Ecuador

Unkuch, Sayda y Rodrigues de Caires, Carlos (2017). Gobernanza e instrumentos de política: un análisis de la política de turismo comunitario del Ecuador. Revista Latinoamericana de Políticas y Acción Pública, Volumen. 4, $\mathrm{N}^{\circ} 2$. Ecuador (Pp. 59-83).

Vásquez, Luis y Manzanilla, Lorenzo (2018).Administración estratégica de empresas turísticas comunitarias con el enfoque de la Teoría de recursos y capacidades en los pueblos indígenas de México. Congreso Internacional de Contaduría, Administración e Informática. Extraído de http://congreso.investiga.fca.unam.mx/docs/xxiii/docs/16.01.pdf consulta $10 / 06 / 19$.

Wernerfelt, Birger (1984). A resource-based view of the firm. Strategic Management Journal. Volumen 5, No 2. Estados Unidos de Norteamérica (Pp. 171-180).

West, Page y Bamford, Charles (2005). Creating a technology-based entrepreneurial economy: A resource based theory perspective. Journal of Technology Transfer. Volumen 30, No 4. Estados Unidos de Norteamérica (Pp 433-451).

Zahra, Shaker; Gedajlovic, Eric; Neubaum, Donald y Shullman, Joel (2009). A typology of social entrepreneurs: Motives, search, processes and ethical challenges. Journal of business venturing. Volumen 24, No 5. Países Bajos (Pp. 519-532). 